\title{
UNUSUAL PRESENTATION OF TAKAYASU ARTERITIS AS DILATED CARDIOMYOPATHY IN YOUNG MALE.
}

\author{
Dolly Joseph, Prakash Joshi, Pranay Bajpai, Padmnabh Sharma, R. K. Jha
}

1. Associate Professor, Department of Medicine, Shri Aurobindo institute of Medical Sciences \& P G Institute.
2. Associate Professor, Department of Medicine, Shri Aurobindo institute of Medical Sciences \& P G Institute.
3. Post Graduate Resident, Department of Medicine, Shri Aurobindo institute of Medical Sciences \& P G
Institute.
4. Post Graduate Resident, Department of Medicine, Shri Aurobindo institute of Medical Sciences \& P G
Institute.
5. Professor \& HOD, Department of Medicine, Shri Aurobindo institute of Medical Sciences \& P G Institute.

\section{CORRESPONDING AUTHOR}

Dr. Dolly Joseph.

Department of Medicine.

Sri Aurbindo Institute of Medical Sciences and PG Institute,

Indore Ujjain state highway, Ujjain ,Madhya Pradesh

E-mail: shaji_dolly22@yahoo.co.in

Ph: 00919826755137

ABSTRACT: Takayasu arteritis is a rare granulomatous systemic disease characterized by stenosis or obliteration of large \& medium sized arteries mainly aorta and its main branches as well as pulmonary artery. Although this disease has variable presentation, but its presentation as Dilated cardiomyopathy is rare. Renal involvement is also less frequently reported in young males. We report a case of $20 \mathrm{yr}$ old male who presented with features of congestive heart failure, renal failure and incidentally detected with absent right radial pulse and hypertension .His CT angiography revealed occlusion of right subclavian artery, superior mesenteric artery \& bilateral renal arteries with splenic infarct suggestive of Takayasu arteritis.

This case report illustrates a rare case of dilated cardiomyopathy and renovascular hypertension with renal failure secondary to Takayasu Arteritis which responded with systemic corticosteroids.

KEY WORDS: Absent pulse, Heart Failure, Malignant Hypertension, Renal failure.

INTRODUCTION: Takayasu arteritis (TA) is a rare chronic inflammatory vasculitis that affects primarily the young female in $2^{\text {nd }}$ and $3^{\text {rd }}$ decade .It occurs worldwide with wide geographical variation, seen mainly in Japan, South East Asia, India and Mexico [1].

It mainly affects the aorta \& its main branches and has variable and nonspecific clinical presentation due to vessel inflammation leading to wall thickening, fibrosis, stenosis, thrombosis, end organ ischemia \& aneurysm formation thus leading to delayed diagnosis and treatment. It is the common cause of Reno vascular hypertension [2].Carotid intimal thickness, Creactive protein, \& elevated ESR are important marker for disease activity, which is followed up in TA [3]. We report a rare case of Takayasu arteritis presented as dilated cardiomyopathy and Reno vascular hypertension

CASE REPORT: A 20 year young male admitted with history of low grade fever and progressive shortness of breath for 20 days, which rapidly worsened to orthopnoea (N Y HA class iv) in one week with severe upper abdominal pain .There was no history of joint pains, limb pain on 
walking (claudication), contact with tuberculosis, jaundice, diabetes mellitus, hypertension or any cardiac disease in past. On physical examination he was tall thin built, febrile, tachypnoeic with respiratory rate of $36 / \mathrm{min}$. His pulse was $108 / \mathrm{min}$ in left radial and absent pulses in right upper limb both brachial \& radial. Lower limb and common carotid pulsations were equal on both the sides. Blood pressure in left upper limb was210/150 $\mathrm{mm}$ of $\mathrm{Hg}$, not recordable in right upper limb \& was equal $(230 / 160 \mathrm{~mm}$ of $\mathrm{Hg})$ in lower limb. He had signs of volume overload like raised JVP, facial puffiness, bilateral pitting pedal edema and tender hepatomegaly. Respiratory system examination revealed fine bibasilar crepitations upto mid scapular area. On auscultation of cardiovascular system $\mathrm{S}_{3}$ gallop, and pansystolic murmur was present in mitral area. Right subclavian bruit and bilateral renal bruit was noted .Ophthalmological examination show normal visual acquity with mild pallor of optic disc.

ON INVESTIGATIONS: He was anemic (hemoglobin 8.6gm\%, normocytic normochromic). Urinalysis revealed microscopic hematuria (6-8 RBC/hpf) \& proteinuria (3+) though his $24 \mathrm{hr}$ urinary protein was $5 \mathrm{mg} / \mathrm{l}$. Renal function tests were deranged with urea $165 \mathrm{mg} / \mathrm{dl} \&$ s. creatinine of $2.75 \mathrm{mg} / \mathrm{dl}$ which increased up to $295 \mathrm{mg} / \mathrm{dl} \& 7.7 \mathrm{mg} / \mathrm{dl}$ respectively. His CRP $(1.2 \mathrm{mg} / \mathrm{dl})$, corrected ESR (26) were elevated. His ANA, HIV, HB $\mathrm{SAG}$, VDRL, MOUNTEX TEST and ASO titer were negative. Serum electrolytes \& Liver function tests were also normal .X-ray chest showed- cardiomegaly (CT RATIO: 65\%) \& pulmonary edema (Fig-1) Echocardiography with color Doppler: showed global hypokinesia with moderate LV dysfunction (LVEF 25\%), moderate (GRADE II) mitral regurgitation and aortic regurgitation \& trivial tricuspid regurgitation, suggestive of dilated cardiomyopathy. USG abdomen revealed bilateral renal parenchymal disease (grade I) and RENAL DOPPLER showed tardus parvus flow in main renal \& interlobar arteries bilaterally (Fig-2.) CT ANGIOGRAPHY revealed occlusion of right subclavian artery with distal flow through collateral, narrowing of proximal part of left subclavian artery with good distal flow, irregular wall thickening of thoracic \& abdominal aorta with focal ring like narrowing of abdominal aorta (50\%), significant right renal artery narrowing at its origin \& occlusion of proximal part of left renal artery and splenic infarct involving significant part of spleen. (Fig 3-4)

Patient was treated initially for congestive heart failure and renal failure with furosemide, digoxin and hemodialysis. Based on clinical findings and supportive investigations diagnosis of vasculitic syndrome-Takayasu arteritis was made and prednisolone in the dose of $1 \mathrm{mg} / \mathrm{kg} /$ day together with antihypertensives was started. On discharge his BP was well controlled, s. creatinine came down from 7.76 to $1.86 \mathrm{mg} / \mathrm{dl}$, and urinary sediments disappeared. Surgery and other interventions were not done as his inflammatory markers were on higher side. On follow up after 2 months his renal function tests returned to normal \& cardiac function improved markedly with LVEF of $45 \%$ in Echocardiography.

DISCUSSION: Takayasu arteritis is a chronic progressive inflammatory disease of large \& medium sized arteries seen mostly in Asian and Hispanic origin with female preponderance. It was first reported by Japanese Ophthalmologist Mikito Takayasu (1908) in a young female with retinal changes. Later Shimzu and Sane (1928) described it as "pulse less disease". Females are affected more commonly than males, with varying ratio as high as 1:9 in Japan to as low as 1:1.2 in Israel where as recent series reported

ratio of 1:6.4 in India ${ }^{[1]}$ In adults TA usually involves aortic arch while in children abdominal aorta is most commonly affected. In Japan proximal aortic involvement with features of 
"reverse coarctation" is seen, where as abdominal aorta involvement with Reno vascular lesion(middle aortic syndrome) is seen in Southeast Asia and Africa [4] .In India Takayasu arteritis as cause of Reno vascular hypertension is reported in 28\%-75\%, Congestive heart failure in $76 \%$, aortic regurgitation in $20-24 \%$ and dilated cardiomyopathy only in 5\%.[5].The presentation as dilated cardiomyopathy is rarely reported and is due to involvement of coronary artery Severe hypertension and cardiac failure are bad prognostic factors.[6-8]

Our case is a young male presented with Reno vascular hypertension, congestive heart failure and dilated cardiomyopathy with absent arterial pulsation in right upper limb. According to ACR (1990) criteria [9] presence of three out of six criteria is required for the diagnosis and our case fulfilled FIVE criteria. It was angiographically confirmed as type $\mathrm{V}$ takayasu arteritis

(According to new angiographic classification of Takayasu arteritis 1994). The incidence of type V TA as reported by Panja et al in largest series is only $10 \%$. ${ }^{11]}$

CONCLUSION: With this case report we want to emphasize that any young patient with heart failure and/or renal failure should be screened for systemic vasculitis, since only an antiinflammatory approach can avoid or at least reduce other complication of vasculitis which can be devastating.

\section{REFERENCES:}

1. Panja M,Mondal PC Current status of Aortoarteritis in India JAPI 2004:VOL52:48-51

2. Sharma BK, Sagar S, Singh AP, Suri S. Takayasu Arteritis in India. Heart Vessels Suppl. 1992; 7:37-43

3. Panico MDB,spichler et al Takayasu arteritis :clinical and therapeutic aspect in 36 patients J.vasc.bras.vol.7 no.2 Porto Alegre June 2008

4. Sen PK, Kinare SG, Engineer SD, Parukar GB. The middle aortic syndrome, Br Heart J 1963;35:610

5. Jain S, Kumari S, Ganguly NK, Sharma BK. Current status of Takayasu Arteritis in India. Int J Cardiol. 1996; 54 Suppl: S111-S116

6. .Kim GB ,Kwon BS, Bae EJ, Noh CI .Takayasu arteritis presenting as dilated cardiomyopathy with left ventricular thrombus in association with ulcerative colitis J Am Coll Cardiol. 2012 Oct 2;60(14):e25

7. V Agrawal, PK Roy, B Bhatia Takayasu arteritis with mid aortic dysplastic syndrome presenting as dilated cardiomyopathy.Journal of Indian College of Cardiology, Volume 2, Issue 1, Pages 40-42 February 2012

8. Ghosh SK, Sinha DP, Ghosh S, Mitra D, Kar AK, Panja M.Dilated cardiomyopathy in nonspecific aortoarteritis. Indian Heart J 1999;51:527-31 


\section{CASE REPORT}

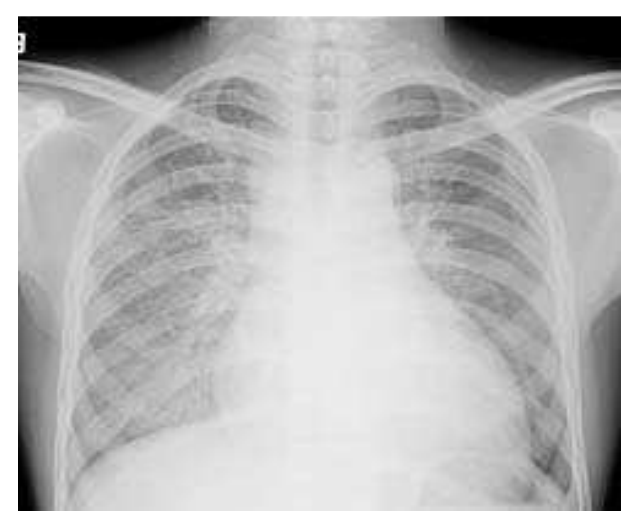

Fig 1 x-ray chest showing cardiomegaly \& pulmonary edema

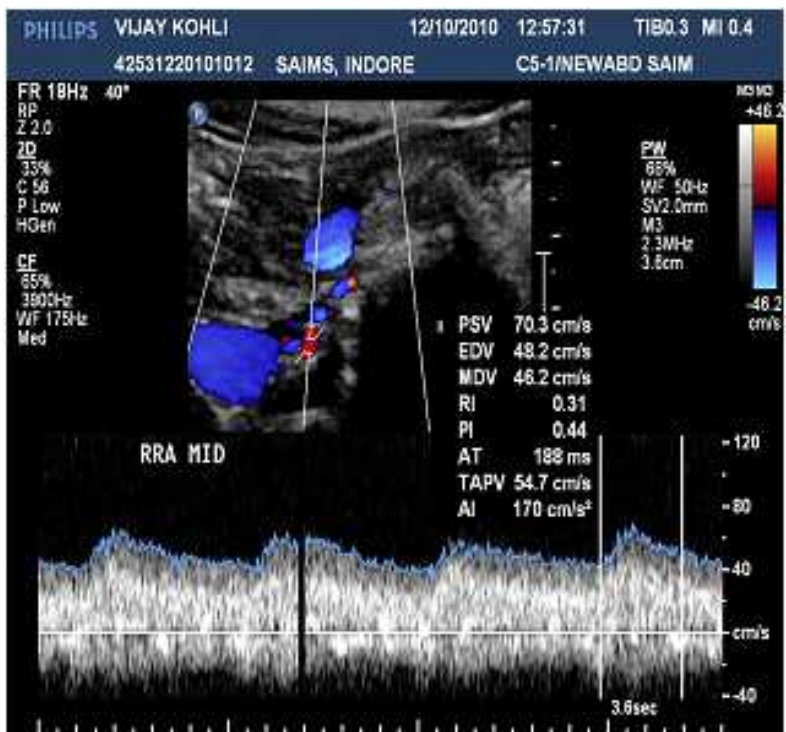

Fig- 2 On spectral Doppler, Right renal artery shows dampening of systolic flow with increase acceleration time suggesting tardus and parvus type flow

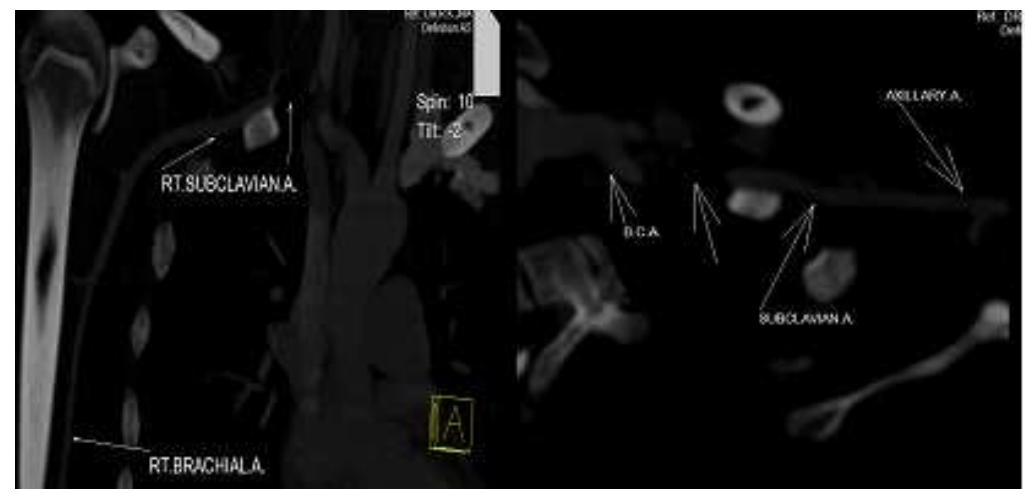

Fig- 3 Occlusion of right (complete) and left (70\%) subclavian artery 


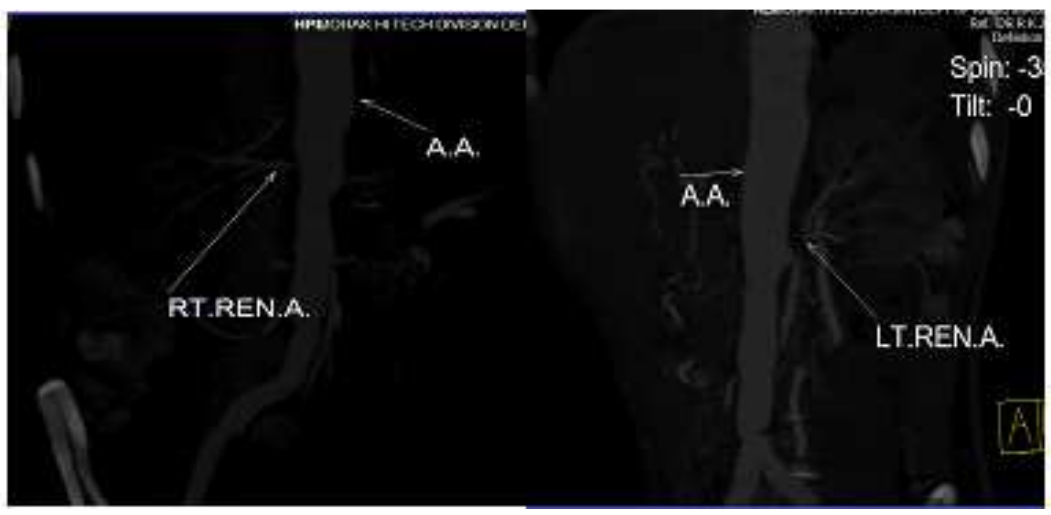

Fig -4 Focal significant narrowing of renal arteries at its origin 